\title{
Disorder-Induced Shift of Condensation Temperature for Dilute Trapped Bose Gases
}

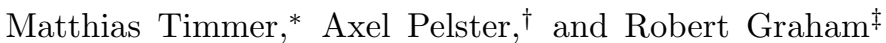 \\ Universität Duisburg-Essen, Campus Essen, Fachbereich Physik, Lotharstrasse 1, 47048 Duisburg, Germany
}

(Dated: September 11, 2018)

\begin{abstract}
We determine the leading shift of the Bose-Einstein condensation temperature for an ultracold dilute atomic gas in a harmonic trap due to weak disorder by treating both a Gaussian and a Lorentzian spatial correlation for the quenched disorder potential. Increasing the correlation length from values much smaller than the geometric mean of the trap scale and the mean particle distance to much larger values leads first to an increase of the positive shift to a maximum at this critical length scale and then to a decrease.

PACS numbers: 03.75.Hh,64.60.Cn
\end{abstract}

The experimental realization of Bose-Einstein condensates (BECs) in magnetically trapped dilute atomic vapors [1, 2] has renewed the theoretical interest in the phase structure of ultracold Bose gases. In particular, many research groups have investigated how the transition temperature $T_{c}$ changes due to a weak local repulsive two-particle interaction $V^{(\mathrm{int})}(\mathbf{x})=4 \pi a / M \delta(\mathbf{x})$ in different trap configurations. Here $a$ denotes the s-wave scattering length, $M$ the atomic mass, and we employ units with $k_{B}=\hbar=1$. Theoretically most demanding is the homogeneous case [3] where the Bose gas of particle density $n$ resides in an infinitely wide trap with a flat potential. There the leading shift $\Delta T_{c} / T_{c}^{(0)}=c_{1} a n^{1 / 3}$ with respect to the interaction-free critical temperature $T_{c}^{(0)}=2 \pi[n / \zeta(3 / 2)]^{2 / 3} / M$ is completely due to longwavelength, critical fluctuations that have to be described nonperturbatively. It can be shown that this shift follows from the classical limit of many-body theory, where only the zero Matsubara modes of the Bose fields are included [4]. A self-consistent variational resummation [5] of the resulting infrared divergent series for $c_{1}$ yields the value $1.27 \pm 0.11[\underline{6}]$ which agrees with the Monte Carlo calculations $1.32 \pm 0.02$ [7, 8]. In contrast to that, harmonic trapping potentials remove the critical long-wavelength fluctuations and reduce the fraction of atoms taking part in nonperturbative physics at the transition point. As a result, the leading shift $\Delta T_{c} / T_{c}^{(0)}=d_{1} a / \lambda_{c}^{(0)}$ in the critical temperature with respect to its noninteracting value $T_{c}^{(0)}=\left[N \omega_{\mathrm{g}} / \zeta(3)\right]^{1 / 3}$ is negative and can be calculated by simple perturbative methods, for instance a Hartree-Fock mean-field approximation [9]. Here $\lambda_{c}^{(0)}=\sqrt{2 \pi /\left[T_{c}^{(0)} M\right]}$ denotes the thermal wavelength and $N$ the boson number, whereas $\omega_{\mathrm{g}}=\left(\omega_{x} \omega_{y} \omega_{z}\right)^{1 / 3}$ is the geometric mean of the respective trap frequencies. The analytically known constant $d_{1}$ has the numerical value $d_{1}=-3.426$ which could be confirmed in a recent experiment within the error bars [10]. Note that a theoretical investigation of the crossover between both physically distinct cases of the homogeneous and the trapped Bose gas has been elaborated in Ref. [11] with the help of general power-law potentials. Further- more, corrections to the leading interaction-induced shift in the critical temperature have been investigated in the Refs. 12, 13, 14, 15].

Apart from the contact interaction, another interesting physical parameter for ultracold atomic vapors is frozen disorder. It can be created artificially by laser speckles 16, 17, 18, 19], incommensurate lattices 20], or different localized atomic species [21, 22]. However, random potentials can also arise naturally via the spatial fluctuations of the electric currents generating the magnetic wire traps [23, 24]. Thus, one can raise the fundamental question how the Bose-Einstein condensation temperature is affected by weak disorder. To this end we consider the quite commonly employed model of a Gaussian distributed disorder potential $V(\mathbf{x})$ with the ensemble averages $\overline{V(\mathbf{x})}=0$ and $\overline{V(\mathbf{x}) V\left(\mathbf{x}^{\prime}\right)}=R\left(\mathbf{x}-\mathbf{x}^{\prime}\right)$. In particular, we are interested in correlation functions $R\left(\mathbf{x}-\mathbf{x}^{\prime}\right)$ which decay with a characteristic correlation length $\xi$. A typical example for such a correlation function is a Lorentzian distribution, which occurs in atom chips [25], more complicated correlation functions can be designed by using laser speckles [26]. Note that the latter experiments allow to tune the corresponding correlation length $\xi$ in a wide parameter range by changing, for instance, the distance between the scattering surface and the magnetooptical trap. So far, the disorder-induced shift of the critical temperature has only been analyzed for a homogeneous Bose gas in the limit $\xi \rightarrow 0$ of a short-range correlation $R(\mathbf{x})=R \delta(\mathbf{x})$. A perturbative calculation of Lopatin and Vinokur [27], motivated by studying the superfluid motion of helium in porous media such as Vycor [28, 29, 30], yields

$$
\frac{\Delta T_{c}}{T_{c}^{(0)}}=-\frac{M^{2} R}{3 \pi[\zeta(3 / 2)]^{2 / 3} n^{1 / 3}} .
$$

The negative sign for the homogeneous Bose gas is compatible with the fact that disorder leads to an effective attractive interaction when the replica method is applied 31.

The purpose of this note is to determine the leading disorder-induced shift in the critical temperature for a weakly interacting Bose gas in an external harmonic 
trap $U(\mathbf{x})$ with an additional weak disorder potential which is characterized by an arbitrary correlation function $R\left(\mathbf{x}-\mathrm{x}^{\prime}\right)$. According to our introduction we expect that disorder leads to an opposite sign of the shift of the critical temperature than repulsive interaction. Since disorder appears within the replica method as an effective attractive interaction [31], the particle density in the center of a harmonic trap is increased. Thus, there should be a positive disorder-induced shift for such a harmonic confinement. Moreover, we should be able to determine it reliably by a perturbative calculation. In first-order perturbation theory, the effects of both interaction and disorder are additive. Therefore, we start with the imaginary-time action of a non-interacting Bose gas in both a trap and a random potential in standard notation

$$
\begin{aligned}
\mathcal{A}\left[\psi^{*}, \psi\right]= & \int_{0}^{\beta} d \tau \int d^{3} x \psi^{*}(\mathbf{x}, \tau)\left[\frac{\partial}{\partial \tau}-\frac{1}{2 M} \boldsymbol{\Delta}\right. \\
& +U(\mathbf{x})+V(\mathbf{x})-\mu] \psi(\mathbf{x}, \tau)
\end{aligned}
$$

and treat the disorder potential $V(\mathbf{x})$ perturbatively. We aim at determining the particle number $N=-\partial \Omega / \partial \mu$ from the grand-canonical free energy $\Omega=-\overline{\ln \mathcal{Z}} / \beta$, where the partition function is determined by the functional integral

$$
\mathcal{Z}=\oint \mathcal{D} \psi^{*} \oint \mathcal{D} \psi e^{-\mathcal{A}\left[\psi^{*}, \psi\right]} .
$$

For an ideal Bose gas a semiclassical treatment of the trap potential leads to

$$
\Omega^{(0)}=\frac{-1}{\beta \lambda^{3}} \int d^{3} x \zeta_{5 / 2}\left(e^{\beta[\mu-U(\mathbf{x})]}\right),
$$

where $\zeta_{a}(z)=\sum_{n=1}^{\infty} z^{n} / n^{a}$ is the polylogarithmic function. Correspondingly, the first-order correction reads

$$
\begin{gathered}
\Omega^{(1)}=-\frac{1}{2 \beta} \int_{0}^{\beta} d \tau_{1} \int_{0}^{\beta} d \tau_{2} \int d^{3} x_{1} \int d^{3} x_{2} \\
\times R\left(\mathbf{x}_{1}-\mathbf{x}_{2}\right) G^{(0)}\left(\mathbf{x}_{1}, \tau_{1} ; \mathbf{x}_{2}, \tau_{2}\right) G^{(0)}\left(\mathbf{x}_{2}, \tau_{2} ; \mathbf{x}_{1}, \tau_{1}\right),
\end{gathered}
$$

where the bosonic Green function has the semiclassical Fourier-Matsubara decomposition

$$
\begin{gathered}
G^{(0)}\left(\mathbf{x}_{1}, \tau_{1} ; \mathbf{x}_{2}, \tau_{2}\right)=\frac{1}{\beta} \sum_{m=-\infty}^{\infty} \int \frac{d^{3} k}{(2 \pi)^{3}} \\
\times e^{-i \omega_{m}\left(\tau_{1}-\tau_{2}\right)+i \mathbf{k}\left(\mathbf{x}_{1}-\mathbf{x}_{2}\right)} G^{(0)}\left(\omega_{m}, \mathbf{k} ; \frac{\mathbf{x}_{1}+\mathbf{x}_{2}}{2}\right)
\end{gathered}
$$

with $G^{(0)}\left(\omega_{m}, \mathbf{k} ; \mathbf{x}\right)=\left[-i \omega_{m}+\mathbf{k}^{2} / 2 M+U(\mathbf{x})-\mu\right]^{-1}$, $\mathbf{x}=\left(\mathbf{x}_{1}+\mathbf{x}_{2}\right) / 2$, and $\omega_{m}=2 \pi m / \beta$. Evaluating in (5) the Matsubara series with the Poisson summation formula [33] and the Fourier integrals with the formula of Plemelj
[34], we obtain

$$
\begin{aligned}
& \Omega^{(1)}=\left(\frac{M}{2 \pi}\right)^{3} \sum_{n=1}^{\infty} \int d^{3} x e^{\beta n[\mu-U(\mathbf{x})]} \int d^{3} x^{\prime} R\left(\mathbf{x}^{\prime}\right) \lim _{\epsilon \searrow 0} \\
& \operatorname{Im} \int_{0}^{\infty} d \tau \frac{e^{-\epsilon \tau}}{[\tau(\tau+i \beta n)]^{3 / 2}} \exp \left\{-\frac{M \beta n \mathbf{x}^{\prime 2}}{2 \tau(\tau+i \beta n)}\right\} .
\end{aligned}
$$

This disorder correction to the grand-canonical free energy is now specialized for a harmonic trap $U(\mathbf{x})$ and for typical correlation functions $R(\mathbf{x})=$ $\int d^{3} k /(2 \pi)^{3} e^{i \mathbf{k x}} R(\mathbf{k})$. For instance, in case of a Gaus$\operatorname{sian} R_{\mathrm{G}}(\mathbf{k})=R e^{-\mathbf{k}^{2} \xi^{2} / 2}$ and a Lorentzian $R_{\mathrm{L}}(\mathbf{k})=$ $R /\left(1+\mathbf{k}^{2} \xi^{2}\right)$, we find

$$
\Omega_{\mathrm{G}}^{(1)}=-\frac{\beta R}{2(2 \pi)^{3 / 2}\left(\beta \omega_{\mathrm{g}}\right)^{3} \xi} \sum_{n=1}^{\infty} \frac{e^{n \beta \mu}}{n^{2}\left(\xi^{2}+\frac{\beta n}{4 M}\right)}
$$

and, correspondingly,

$$
\begin{aligned}
\Omega_{\mathbf{L}}^{(1)}= & -\frac{\pi \sqrt{M \beta} R}{2(2 \pi)^{3 / 2} \xi^{2}\left(\beta \omega_{\mathrm{g}}\right)^{3}} \sum_{n=1}^{\infty} \frac{e^{\beta n \mu}}{n^{5 / 2}} \\
& \times \exp \left[\frac{\beta n}{8 M \xi^{2}}\right]\left[1-\Phi\left(\sqrt{\frac{\beta n}{8 M \xi^{2}}}\right)\right],
\end{aligned}
$$

where we have used for the latter the probability integral $\Phi(z)=2 / \sqrt{\pi} \int_{0}^{z} d t e^{-t^{2}}$.

As follows from the theory of critical phenomena [35, 36], the phase transition to a Bose-Einstein condensate occurs when the spatio-temporal integral over the correlation function

$G\left(\mathbf{x}_{1}, \tau_{1} ; \mathbf{x}_{2}, \tau_{2}\right)$

$$
=\overline{\frac{1}{\mathcal{Z}} \oint \mathcal{D} \psi^{*} \oint \mathcal{D} \psi \psi\left(\mathbf{x}_{1}, \tau_{1}\right) \psi^{*}\left(\mathbf{x}_{2}, \tau_{2}\right) e^{-\mathcal{A}\left[\psi^{*}, \psi\right]}}
$$

diverges, i.e. $G\left(\omega_{m}, \mathbf{k} ; \mathbf{x}\right)$ becomes infinite for $\omega_{m}=0$, $\mathbf{k}=\mathbf{0}$, and some $\mathbf{x}$. This criticality criterion is equivalent to the vanishing of its inverse

$$
G^{-1}(0, \mathbf{0} ; \mathbf{x})=G^{(0)-1}(0, \mathbf{0} ; \mathbf{x})-\Sigma(0, \mathbf{0} ; \mathbf{x}),
$$

where the first-order perturbative contribution to the self-energy is given by

$$
\Sigma^{(1)}\left(\mathbf{x}_{1}, \tau_{1} ; \mathbf{x}_{2}, \tau_{2}\right)=R\left(\mathbf{x}_{1}-\mathbf{x}_{2}\right) G^{(0)}\left(\mathbf{x}_{1}, \tau_{1} ; \mathbf{x}_{2}, \tau_{2}\right) .
$$

Therefore, we define the critical chemical potential $\mu_{c}$ from the condition

$$
\underset{\mathbf{x}}{\operatorname{Min}} G^{-1}(0, \mathbf{0} ; \mathbf{x})=0 .
$$

Up to first order in the disorder we obtain a result which does not depend on the trap frequencies:

$$
\mu_{c}^{(1)}=-2 M \int d^{3} x \frac{R(\mathbf{x})}{|\mathbf{x}|} .
$$




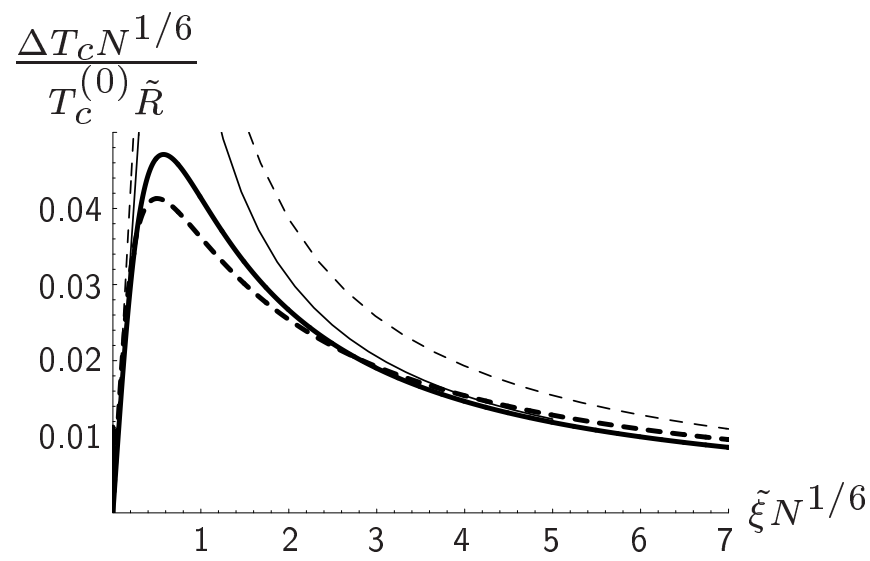

FIG. 1: Disorder-induced shifts (15) and (16) of the critical temperature $\Delta T_{c}$ with respect to the interaction-free critical temperature $T_{c}^{(0)}$ for the Gaussian (solid) and the Lorentzian (dashed) correlation function together with their asymptotics (17) and (18) as a function of the dimensionless correlation length.

We conclude from (14) that Bose-Einstein condensation occurs with disorder earlier than in the clean case. Note that the critical chemical potential for the onset of BoseEinstein condensation follows from condition (13) also in other physical situations, for instance, when anisotropic traps are used for rotating Bose gases [37] or when dipolar gases are considered where the two-particle interaction contains both a short-range, isotropic contact potential and a long-range, anisotropic dipole-dipole interaction [38, 39].

Now we combine our previous results and determine the disorder-induced shift in the critical temperature. To this end we evaluate $N=N(\mu)$ in the limit $\mu \uparrow \mu_{c}$ by taking into account the Robinson formula [40] for the polylogarithmic function in (4). For the Gaussian correlation we find

$$
\begin{aligned}
\frac{\Delta T_{c, \mathrm{G}}}{T_{c}^{(0)}}= & \frac{8 \tilde{R} \tilde{\xi}}{3(2 \pi)^{3 / 2} \zeta(3)} \\
& \times \sum_{k=1}^{\infty} \frac{1}{k^{2}\left[k+4 N^{1 / 3} \tilde{\xi}^{2} /[\zeta(3)]^{1 / 3}\right]},
\end{aligned}
$$

whereas we obtain for the Lorentzian correlation

$$
\begin{aligned}
& \frac{\Delta T_{c, \mathrm{~L}}}{T_{c}^{(0)}}=\frac{\tilde{R}}{6 \pi[\zeta(3)]^{2 / 3} \tilde{\xi} N^{1 / 3}} \sum_{k=1}^{\infty}\left\{\frac{1}{k^{2}}-\frac{[\zeta(3)]^{1 / 6} \sqrt{\pi}}{\sqrt{8} \tilde{\xi} N^{1 / 6} k^{3 / 2}}\right. \\
& \left.\quad \times \exp \left[\frac{[\zeta(3)]^{1 / 3} k}{8 \tilde{\xi}^{2} N^{1 / 3}}\right]\left[1-\Phi\left(\sqrt{\frac{[\zeta(3)]^{1 / 3} k}{8 \tilde{\xi}^{2} N^{1 / 3}}}\right)\right]\right\} .
\end{aligned}
$$

Here we have introduced dimensionless units for the correlation length $\tilde{\xi}=\xi / l_{\text {os }}$ as well as for the disorder strength $\tilde{R}=M^{2} l_{\mathrm{os}} R$, where $l_{\mathrm{os}}=1 / \sqrt{M \omega_{\mathrm{g}}}$ denotes the oscillator length. Note that both disorder-induced

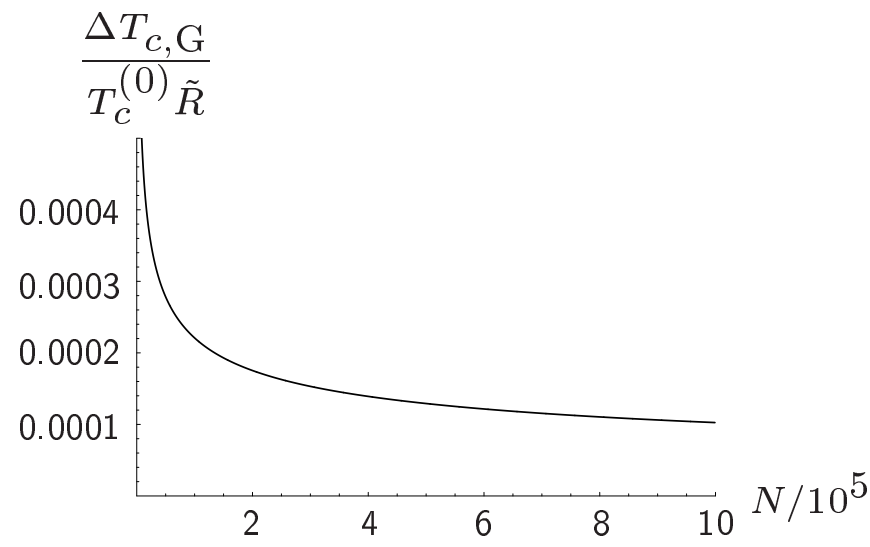

FIG. 2: Normalized shift of the critical temperature $\Delta T_{c, \mathrm{G}} /\left(T_{c}^{(0)} \tilde{R}\right)$ of a dilute ${ }^{85} \mathrm{Rb}$ gas in an anisotropic harmonic trap with geometric mean frequency $\omega_{\mathrm{g}}=2 \pi \cdot 40 \mathrm{~Hz}$ and a Gaussian disorder correlation with correlation length $\xi=10 \mu \mathrm{m}$ versus particle number $N$.

shifts (15) and (16) have similar asymptotics in the limit $\xi \rightarrow 0$

$$
\frac{\Delta T_{c, \mathrm{G}}}{T_{c}^{(0)}} \approx \frac{8 \tilde{R} \tilde{\xi}}{3(2 \pi)^{3 / 2}}, \quad \frac{\Delta T_{c, \mathrm{~L}}}{T_{c}^{(0)}} \approx \frac{2 \tilde{R} \tilde{\xi}}{3 \pi}
$$

and in the limit $\xi \rightarrow \infty$

$$
\begin{aligned}
\frac{\Delta T_{c, \mathrm{G}}}{T_{c}^{(0)}} & \approx \frac{2 \zeta(2) \tilde{R}}{3(2 \pi)^{3 / 2}[\zeta(3)]^{2 / 3} \tilde{\xi} N^{1 / 3}}, \\
\frac{\Delta T_{c, \mathrm{~L}}}{T_{c}^{(0)}} & \approx \frac{\zeta(2) \tilde{R}}{6 \pi[\zeta(3)]^{2 / 3} \tilde{\xi} N^{1 / 3}} .
\end{aligned}
$$

Figure 1 shows how the shift results (15) and (16) vary with the dimensionless correlation length $\tilde{\xi}$. Whereas for small $\tilde{\xi}$ the oscillator length $l_{\text {os }}$ dominates, the average particle distance $l_{\mathrm{p}}=l_{\mathrm{os}} / N^{1 / 3}$ becomes important for large $\tilde{\xi}$. Both length scales meet at $\sqrt{l_{\mathrm{os}} l_{\mathrm{p}}}=l_{\mathrm{os}} / N^{1 / 6}$, i.e. $\tilde{\xi} N^{1 / 6}=1$, which is roughly where the maximal shift occurs. This result will certainly help to design an experiment which aims at detecting a disorder-induced shift of the critical temperature.

Furthermore, it is clear from (15), (16), and Figure1 that the shifts for Gaussian or Lorentzian spatial correlations are qualitatively and even semi-quantitatively the same. This finding justifies to apply our results also to other correlation functions, which decay with a characteristic correlation length $\xi$ as, for instance, the laser speckle correlations in the Florence experiment [16, 18]. There about $N=3 \cdot 10^{5}$ atoms of the ${ }^{85} \mathrm{Rb}$ isotope reside in an anisotropic harmonic trap with the geometric mean frequency $\omega_{\mathrm{g}}=2 \pi \cdot 40 \mathrm{~Hz}$, so that its oscillator length is $l_{\mathrm{os}}=1.72 \mu \mathrm{m}$. The disorder potential is characterized by a length scale $\xi=10 \mu \mathrm{m}$, i.e. $\tilde{\xi}=5.88$, and a strength $R$ which is measured in units of a frequency $\omega$. As $R$ has 
the physical units (energy) ${ }^{2}$ (length) ${ }^{3}$, the dimensionless disorder strength can be estimated by $\tilde{R}=\left(\omega / \omega_{\mathrm{g}}\right)^{2} \tilde{\xi}^{3}$. A weak disorder potential, which only slightly perturbes the condensate in the Florence experiment, corresponds to the frequency $\omega=2 \pi \cdot 50 \mathrm{~Hz}$, yielding $\tilde{R}=200$. In Figure 2 the normalized shift of the critical temperature $\Delta T_{c} /\left(T_{c}^{(0)} \tilde{R}\right)$ is plotted as a function of the particle number $N$ for a Gaussian correlation, so we conclude that the disorder-induced shift in the critical temperature is in this case of the order of $3 \%$. With this it is about the same size as the interaction-induced shift [9] and should thus be measurable [10]. Furthermore, we read off from Figure 2 that this shift increases with a decreasing number of particles in the Bose gas.

In this letter we have determined the disorder-induced shift of the critical temperature for a Bose gas in a harmonic trap. By treating the analytically solvable cases of a Gaussian and a Lorentzian correlation function, we have estimated that this disorder-induced shift should be measurable in laser speckle experiments. Note that applying our general perturbative results (5) and (14) to a homogeneous Bose gas and to a vanishing correlation length $\xi=0$ reproduces the seminal LopatinVinokur shift (10). However, in this case the interactioninduced shift of the critical temperature suggests that additional nonperturbative contributions to the disorderinduced shift will certainly occur. First indications for this conjecture are provided by a recent momentum-shell renormalization group calculation 32].

This work was supported by the SFB/TR 12 of the German Research Foundation (DFG).

* Electronic address: sp0088@theo-phys.uni-essen.de

† Electronic address: axel.pelster@uni-due.de

¥ Electronic address: robert.graham@uni-due.de

[1] M.H. Anderson, J.R. Ensher, M.R. Matthews, C.E. Wieman, and E.A. Cornell, Science 269, 198 (1995).

[2] K.B. Davis, M.-O. Mewes, M.R. Andrews, N.J. van Druten, D.S. Durfee, D.M. Kurn, and W. Ketterle, Phys. Rev. Lett. 75, 3969 (1995).

[3] J.O. Andersen, Rev. Mod. Phys. 76, 599 (2004).

[4] G. Baym, J.P. Blaizot, M. Holzmann, F. Laloë, and D. Vautherin, Phys. Rev. Lett. 83, 1703 (1999).

[5] H. Kleinert, Mod. Phys. Lett. B 17, 1011 (2003).

[6] B. Kastening, Phys. Rev. A 69, 043613 (2004).

[7] V.A. Kashurnikov, N.V. Prokof'ev, and B.V. Svistunov, Phys. Rev. Lett. 87 120402, (2001).

[8] P. Arnold and G. Moore, Phys. Rev. Lett. 87, 120401 (2001).

[9] S. Giorgini, L.P. Pitaevskii, and S. Stringari, Phys. Rev. A 54, R4633 (1996).

[10] F. Gerbier, J.H. Thywissen, S. Richard, M. Hugbart, P. Bouyer, and A. Aspect, Phys. Rev. Lett. 92, 030405 (2004).

[11] O. Zobay, G. Metikas, and H. Kleinert, Phys. Rev. A 71, 043614 (2005)
[12] P. Arnold, G. Moore, and B. Tomášik, Phys. Rev. A 65, 013606 (2002).

[13] P. Arnold and B. Tomášik, Phys. Rev. A 64, 053609 (2001)

[14] M.J. Davis and P.B. Blakie, Phys. Rev. Lett. 96, 060404 (2006).

[15] Y.-M. Kao and T.F. Jiang, Phys. Rev. A 73, 043604 (2006).

[16] J.E. Lye, L. Fallani, M. Modugno, D.S. Wiersma, C. Fort, and M. Inguscio, Phys. Rev. Lett. 95, 070401 (2005).

[17] D. Clément, A.F. Varón, M. Hugbart, J.A. Retter, P. Bouyer, L. Sanchez-Palencia, D.M. Gangardt, G.V. Shlyapnikov, and A. Aspect, Phys. Rev. Lett. 95, 170409 (2005).

[18] C. Fort, L. Fallani, V. Guarrera, J.E. Lye, M. Modugno, D.S. Wiersma, and M. Inguscio, Phys. Rev. Lett. 95, 170410 (2005).

[19] T. Schulte, S. Drenkelforth, J. Kruse, W. Ertmer, J. Arlt, K. Sacha, J. Zakrzewski, and M. Lewenstein, Phys. Rev. Lett. 95, 170411 (2005).

[20] B. Damski, J. Zakrzewski, L. Santos, P. Zoller, and M. Lewenstein, Phys. Rev. Lett. 91, 080403 (2003).

[21] U. Gavish and Y. Castin, Phys. Rev. Lett. 95, 020401 (2005).

[22] S. Ospelkaus, C. Ospelkaus, O. Wille, M. Succo, P. Ernst, K. Sengstock, and K. Bongs, Phys. Rev. Lett. 96, 180403 (2006).

[23] D.-W. Wang, M. D. Lukin, and E. Demler, Phys. Rev. Lett. 92, 076802 (2004).

[24] T. Schumm, J. Esteve, C. Figl, J.-B. Trebbia, C. Aussibal, H. Nguyen, D. Mailly, I. Bouchoule, C.I. Westbrook, and A. Aspect, Eur. Phys. J. D 32, 171 (2005).

[25] R. Folman, P. Krüger, J. Schmiedmayer, J. Denschlag, and C. Henkel, Adv. At. Mol. Opt. Phys. 48, 263 (2002).

[26] J.C. Dainty (Ed.), Laser Speckle and Related Phenomena (Springer, Berlin, 1975).

[27] A.V. Lopatin and V.M. Vinokur, Phys. Rev. Lett. 88, 235503 (2002).

[28] B.C. Crooker, B. Hebral, E.N. Smith, Y. Takano, and J.D. Reppy, Phys. Rev. Lett. 51, 666 (1983).

[29] M.H.W. Chan, K.I. Blum, S.Q. Murphy, G.K.S. Wong, and J.D. Reppy, Phys. Rev. Lett. 61, 1950 (1988).

[30] K. Huang and H.F. Meng, Phys. Rev. Lett. 69, 644 (1992).

[31] R. Graham and A. Pelster, eprint: cond-mat/0508306

[32] O. Zobay, Phys. Rev. A 73, 023616 (2006).

[33] H. Kleinert, Path Integrals in Quantum Mechanics, Statistics, Polymer Physics, and Financial Markets, Forth Extended Edition (World Scientific, Singapore, 2006).

[34] W. Heitler, The Quantum Theory of Radiation, Third Edition (Oxford University Press, Oxford, 1970).

[35] J. Zinn-Justin, Quantum Field Theory and Critical Phenomena, 3rd Edition (Oxford University Press, Oxford, 1996).

[36] H. Kleinert and V. Schulte-Frohlinde, Critical Properties of $\Phi^{4}$-Theories (World Scientific, Singapore, 2001).

[37] S. Kling and A. Pelster, eprint: cond-mat/0604162

[38] K. Glaum, A. Pelster, H. Kleinert, and T. Pfau, eprint: cond-mat/0606569.

[39] K. Glaum and A. Pelster, eprint: cond-mat/0609374

[40] J.E. Robinson, Phys. Rev. 83, 678 (1951). 